\title{
The prognostic role of Charlson comorbidity index for critically ill elderly patients
}

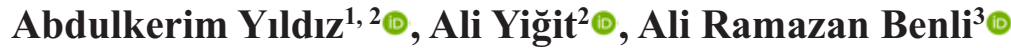 \\ ${ }^{1}$ Department of Hematology, University of Health Sciences, Dışkapı Yıldırım Beyazit, Training and Research Hospital, Ankara, Turkey \\ ${ }^{2}$ Department of Internal Medicine, Karabük University, Karabuk Training and Research Hospital, Karabük, Turkey \\ ${ }^{3}$ Department of Family Medicine, Karabuk University School of Medicine, Karabük, Turkey
}

\section{ABSTRACT}

Objectives: Comorbidities represent a risk factor for adverse events in several critical illnesses. The aim of this study was to identify the relationship between the Charlson Comorbidity Index (CCI) with mortality and length of stay (LOS) in critically ill elderly patients.

Methods: A retrospective analysis was made of patients admitted to our tertiary adult intensive care unit (ICU) between January 2015 and January 2016. The impact of comorbidity was evaluated with the CCI. Other required data were retrieved from the patients' follow-up records.

Results: The study included a total of 251 patients. The mean age was $78.79 \pm 6.70$ years. The total mortality rate was $41.0 \%$. The most common cause for admission was sepsis and acute respiratory failure $(18.3 \%$ vs 18.3\%). The median APACHE II score was significantly higher in non-survivors than survivors (31.0 [13.047.0] vs 21.0 [9.0-40.0]; $p<0.01)$. The median CCI was $2.0(0.0-7.0)$ for survivors and $3.0(1.0-10.0)$ for non-survivors. The CCI of non-survivors was significantly higher than that of survivors $(p=0.005)$. Patients with $\mathrm{CCI}>3$ had higher mortality than those with $\mathrm{CCI} \leq 3(p<0.05)$. The odds ratio of the APACHE II score for mortality was 1.214 (95\% CI: 1.154-1.276), and for CCI it was 1.320 (95\% CI: 1.088-1.602). There was a significant positive correlation between CCI and LOS ( $\mathrm{r}=0.147 ; p=0.020)$.

Conclusions: CCI is strongly associated with both mortality and LOS. It can be used as a prognostic marker for elderly patients in critical care.

Keywords: Charlson comorbidity index, older patients, critically ill, mortality

T here are many prognostic factors and scoring systems for mortality and prognosis in ICU patients. Of these, the Acute Physiology and Chronic Health Evaluation (APACHE) score is one of the most frequently used scoring systems [1]. In recent years, there has been increasing interest in different indicators which may be useful for the prediction of mortality.

Population aging and the increasing frequency of chronic diseases has created a greater need for acute healthcare services, with an increased number of elderly patients admitted to ICU [2]. Comorbidity is one of the major factors affecting health status and management strategies in critically ill patients especially the elderly [3]. The coexistence of two or more longterm pathologies is defined as multimorbidity, and this condition is related to frailty, disability, increased risk of hospitalization and death, especially among elderly patients [4].

The Charlson Comorbidity Index (CCI), first re-

How to cite this article: Yıldız A, Yiğit A, Benli AR. The prognostic role of Charlson comorbidity index for critically ill elderly patients. Eur Res $J$ 2020;6(1):67-72. DOI: 10.18621/eurj.451391

Address for correspondence: .Abdulkerim Yıldız, MD., University of Health Sciences, Dışkapı Yıldırım BeyazitTraining and Research Hospital, Department of Hematology, Ankara,Turkey.E-mail: akerim@hotmail.com,Fax:+90 3122562340 
ported in 1987, is a commonly used index which is used for predicting prognosis based on comorbid conditions of the patient [5]. The CCI was subsequently shown to be important and effective prognostic marker for mortality in many diseases and conditions $[6,7]$. The aim of this study was to present the the prognostic value of the CCI for mortality and length of stay (LOS) in older patients admitted to the Internal Medicine Adult ICU of Karabuk University Training and Research Hospital.

\section{METHODS}

A retrospective analysis was made of patients admitted to the Medical ICU of Karabuk University Training and Research Hospital from January 2015 to January 2016. Patients with a length of stay (LOS) of less than 48 hours and younger than 65 years age were excluded. The study included a total of 251 patients aged $>65$ years.

Evaluation was made of the demographic information, laboratory test results, APACHE II scores, and diagnosis at the time of admission. Comorbidity was defined as pre-existing diseases and medical conditions present at the time of admission. The comorbidities and conditions of the patients were recorded. The CCI score was calculated using these parameters. Table 1 shows the list of comorbid conditions included in the CCI. For the analyses, the study population was separated into 2 groups as survivors and non-survivors.

\section{Compliance with Ethical Standards}

All procedures performed in the study were in accordance with the ethical standards of the institutional and/or national research committee and with the 1964 Helsinki Declaration and its later amendments or comparable ethical standards.

\section{Statistical Analysis}

SPSS Statistics 19 software (IBM, Armonk, NY, USA) was used for statistical analysis. In the comparison of variables distributed homogeneously, the t-test was used for parametric variables and the Chi-Square test was used for non-parametic variables. For variables not showing homogeneous distribution, the Mann Whitney U test was used. Data were expressed as mean \pm standard deviation (SD) and median (minimum-maximum) values. Multiple logistic regression analyses were used to determine the risk factors for mortality and LOS. Kaplan-Meier survival curves was used for survival analysis. A value of $p<0.05$ was accepted as statistically significant.

\section{RESULTS}

The total 251 patients comprised 109 males (43.4\%) and 142 females (56.6\%) with a mean age of $78.79 \pm 6.70$ years. A total of $103(41.0 \%)$ patients died during the follow-up period and 148 (59.0\%) patients survived and were either transferred to other departments in the hospital or discharged. The demographic data are shown in Table 2. The most common diagnoses for ICU admission were acute respiratory failure $(18.3 \%)$, sepsis $(18.3 \%)$ and pneumonia (15.9\%). Median overall survival was 17 days (14.9-19.1) (Fig. 1).

\section{Table 1. Charlson Comorbidity Index}

\begin{tabular}{|c|c|}
\hline Condition or disease & Comorbidity Score \\
\hline Coronary artery disease & 1 \\
\hline \multicolumn{2}{|l|}{ Congestive heart failure } \\
\hline \multicolumn{2}{|l|}{ Peripheral vascular disease } \\
\hline \multicolumn{2}{|l|}{ Cerebrovascular disease } \\
\hline \multicolumn{2}{|l|}{ Dementia } \\
\hline \multicolumn{2}{|l|}{ Chronic pulmonary disease } \\
\hline \multicolumn{2}{|l|}{ Connective tissue disorder } \\
\hline \multicolumn{2}{|l|}{ Peptic ulcer disease } \\
\hline \multicolumn{2}{|l|}{ Mild liver disease } \\
\hline \multicolumn{2}{|l|}{ Diabetes } \\
\hline Hemiplegia & 2 \\
\hline \multicolumn{2}{|l|}{$\begin{array}{l}\text { Moderate or severe renal } \\
\text { disease }\end{array}$} \\
\hline \multicolumn{2}{|l|}{$\begin{array}{l}\text { Diabetes with end-organ } \\
\text { damage }\end{array}$} \\
\hline \multicolumn{2}{|l|}{$\begin{array}{l}\text { Any tumor, leukemia, } \\
\text { lymphoma }\end{array}$} \\
\hline $\begin{array}{l}\text { Moderate or severe liver } \\
\text { disease }\end{array}$ & 3 \\
\hline Metastatic solid tumor & 6 \\
\hline AIDS & \\
\hline
\end{tabular}




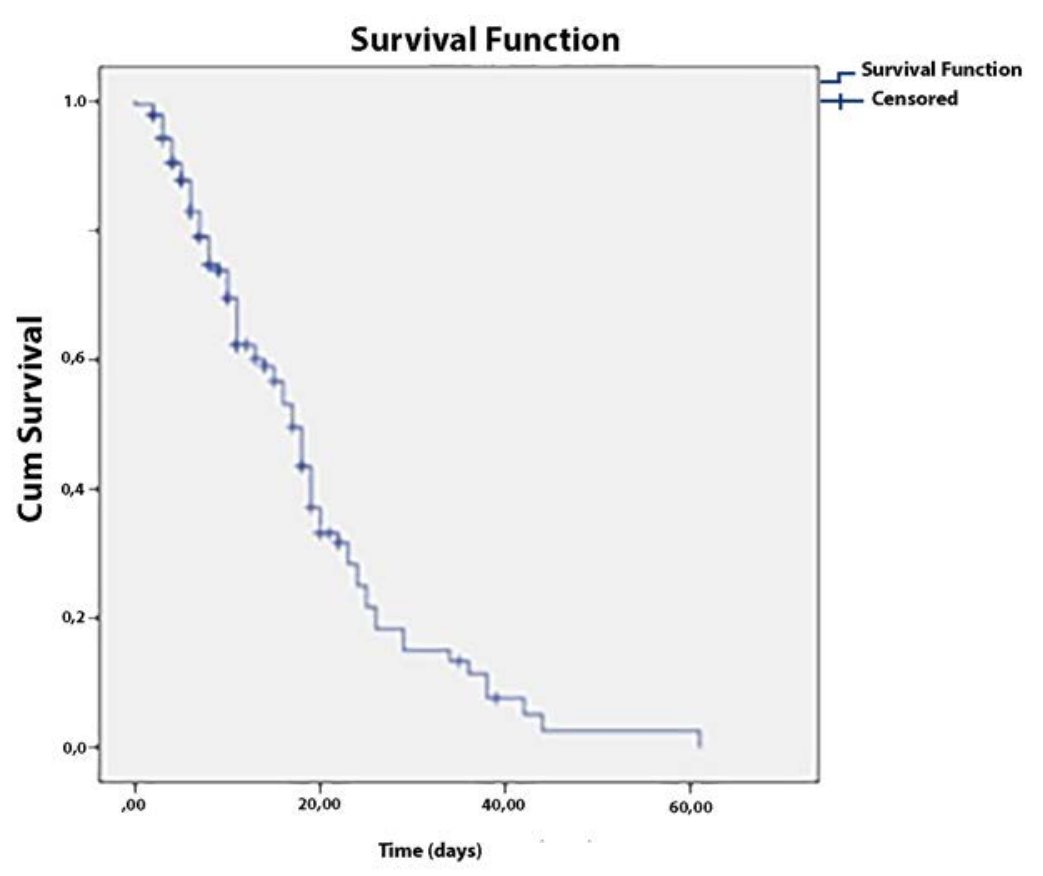

Fig. 1. Kaplan-Meier survial analysis for overall survial of the patients

The median age was 80.0 years $(65.0-93.0)$ for non-survivors, which was higher than that of survivors at 79.5 years (65.0-99.0), but not statistically significant $(p=0.975)$. The median APACHE II score of the whole patient group was 26.0 (9.0-47.0). It was significantly higher in non-survivors than survivors (31.0 [13.0-47.0] vs 21.0 [9.0-40.0]; $p<0.01$ ). The median CCI was $2.0(0.0-7.0)$ for survivors and 3.0 (1.0-10.0) for non-survivors. The CCI of nonsurvivors was significantly higher than that of survivors $(p=0.005)$. All these parameters and the relationships with mortality are shown in Table 3 . The mortality rate of patients with $\mathrm{CCI}>3$ was significantly higher than patients with $\mathrm{CCI} \leq 3(55.1 \%$ vs $35.7 \% ; p<0.05$ ) (Table 4 ).

Multiple logistic regression analysis was applied to the APACHE II score, age and CCI, and the APACHE II and CCI were left on the model with a value of $p<0.491$. According to the result of this analysis, the odds ratio of APACHE II score for mortality was found to be 1.214 (95\% Confidence Interval: 1.154-1.276), and the odds ratio of CCI for mortality was found to be 1.320 (95\% Confidence Interval: 1.088-1.602).

When the relationship between the APACHE II, CCI and LOS was analysed, there was a significant positive correlation between APACHE II and LOS ( $\mathrm{r}$ $=0.314 ; p<0.001)$. CCI was found to be positively related with $\operatorname{LOS}(\mathrm{r}=0.147 ; p=0.020)$. There was no significant relationship between CCI and APACHE II $(p>0.05)$. The relationships between the parameters are shown in Table 5.

\section{DISCUSSION}

Many studies have examined the factors affecting mortality and the risk scoring methods in intensive care patients. Although there are several scoring

Table 2. Demographic characteristics of all patients $(\mathrm{n}=\mathbf{2 5 1})$

\begin{tabular}{|c|c|}
\hline Age (years) & $78.79 \pm 6.70$ \\
\hline \multicolumn{2}{|l|}{ Gender } \\
\hline Male & $109(43.4 \%)$ \\
\hline Female & $142(56.6 \%)$ \\
\hline APACHE II score & $26.0(9.0-47.0)$ \\
\hline CCI & $2.0(0.0-10.0)$ \\
\hline Length of stay (days) & $10.06 \pm 8.60$ \\
\hline \multicolumn{2}{|l|}{ Final status } \\
\hline Survivors & $148(59.0 \%)$ \\
\hline Non-survivors & $103(41.0 \%)$ \\
\hline
\end{tabular}

APACHE $=$ Acute Physiology and Chronic Health Evaluation, $\mathrm{CCI}=$ Charlson Comorbidity Index 


$\begin{aligned} & \text { Table 3. APACHE II score and comorbidity index and their relationship with } \\
& \text { mortality }\end{aligned}$
\begin{tabular}{lccc} 
Parameters & $\begin{array}{c}\text { Survivors } \\
(\mathbf{n}=\mathbf{1 4 8}) \\
\text { Median (range) }\end{array}$ & $\begin{array}{c}\text { Non-survivors } \\
(\mathbf{n}=\mathbf{1 0 3}) \\
\text { Median (range) }\end{array}$ & p value \\
\hline Age & $79.5(65.0-99.0)$ & $80.0(65.0-93.0)$ & 0.975 \\
APACHE II score & $21.0(9.0-40.0)$ & $31.0(13.0-47.0)$ & $<0.001$ \\
CCI & $2.0(0.0-7.0)$ & $3.0(1.0-10.0)$ & 0.005 \\
\hline
\end{tabular}

APACHE: Acute Physiology and Chronic Health Evaluation, CCI: Charlson Comorbidity Index

systems, the APACHE II score is still one of the most frequently used risk scoring systems and it has high success rates for the prediction of mortality [1]. In the current study, the APACHE II score was determined to have a significantly positive correlation with both mortality and LOS, as expected. In a prospective study of 109 patients admitted to the ICU for 1 year, malnutrition, delirium, and APACHE II were found to be risk factors for long-term mortality [8].

Recently, the mean age of patients admitted to ICU has been increasing [2,9]. Aging itself is considered to be the most important risk factor for several chronic conditions [10]. In two studies of elderly patients, age itself was not found to be an independent risk factor for mortality $[8,11]$. In only one study, mortality was significantly associated with age in univariate analysis but not in multivariate analysis [12].

In the current study, the mean age of the patients was $78.79 \pm 6.70$ years and similar to previous results, it was demonstrated that age is not a risk factor for mortality and LOS in older patients. As APACHE II was the already known predictive score and age was shown not to be related with mortality and LOS, then the comorbidity status becomes very important. For elderly patients, there is no generally valuable and validated index for comorbidity which can predict prognosis especially in intensive care.

The Charlson comorbidity index (CCI) is the most extensively studied comorbidity index for predicting

Table 4. The relationship between CCI and mortality

\begin{tabular}{lccc}
\hline & $\begin{array}{c}\text { Survivors } \\
\text { n (\%) }\end{array}$ & $\begin{array}{c}\text { Non-survivors } \\
\text { n (\%) }\end{array}$ & p value \\
\hline Patients with CCI $\leq 3$ & $117(64.3 \%)$ & $65(35.7 \%)$ & $<\mathbf{0 . 0 5}$ \\
Patients with CCI $>3$ & $31(44.9 \%)$ & $38(55.1 \%)$ & \\
Total & $148(59.0 \%)$ & $103(41.0 \%)$ & \\
\hline
\end{tabular}

CCI: Charlson Comorbidity Index

Tablo 5. The relationship between CCI, APACHE II score and length of stay

\begin{tabular}{ccccc}
\hline $\mathbf{( n = 2 5 1 )}$ & & LOS & APACHE II & CCI \\
\hline LOS & r & 1.000 & 0.314 & 0.147 \\
& $p$ & - & $<\mathbf{0 . 0 0 1}$ & $\mathbf{0 . 0 2 0}$ \\
APACHE II & r & $\#$ & 1.000 & 0.108 \\
& $p$ & & - & 0.087 \\
CCI & r & $\#$ & $\#$ & 1.000 \\
& $p$ & & & - \\
\hline
\end{tabular}

APACHE $=$ Acute Physiology and Chronic Health Evaluation, CCI = Charlson Comorbidity Index, LOS $=$ Length of stay 
mortality. It includes the number and severity of comorbid conditions [5]. The CCI has been shown to be important and effective prognostic marker for mortality in many diseases and conditions $[6,7]$, although the number of studies on critically ill elderly patients is limited. In a study of elderly patients admitted to ICU, age, critical illness, cardiopulmonary resuscitation, and the need for mechanical ventilation and/or vasopressor therapy were found to be independent risk factors associated with adverse outcomes [2]. In the current study, the CCI of nonsurvivors was found to be significantly higher than that of survivors and the OR was 1.320 for mortality. Therefore, this finding supports the view that CCI is a valuable index for critically ill elderly patients. Similar to the current study, Buntinx et al. [13] showed that the CCI is a predictor of short-term mortality and hospitalization in a large cohort of 2,624 institutionalized elderly people. In contrast, in a prospective study with 444 elderly patients, a comparison was made of the abilities of six different validated comorbidity indices including $\mathrm{CCI}$, to predict mortality, LOS and institutionalization in a geriatric hospital. The results showed that the geriatrics index of comorbidity (GIC) was the most accurate predictor of mortality during hospitalization rather than CCI [12]. In contrast, the results of the current study demonstrated that both APACHE II and CCI have a significant impact on LOS. There was no relationship between CCI and APACHE II. Therefore it can be suggested that CCI be used as a prognostic marker for LOS in addition to APACHE II.

Quach et al. [14] prospectively compared the discriminative ability of the CCI to the APACHE II in predicting hospital mortality in adult multisystem ICU patients. They found that CCI is a poor predictor of mortality and does not perform as well as the APACHE II in predicting hospital mortality in ICU patients. However, they suggested that CCI can be considered as an alternative method of risk assesment when illness scores are unavailable or are not recorded in a standard way. Since the CCI includes comorbidies which are usually seen in older ages, one of the reasons for their result maybe that they included patients $>17$ years with a median age of 56. In our study, we only included geriatric patients (age $>65$ years) which are expected to have chronic comorbidies before ICU administration. In a prospective cohort study of $201 \mathrm{ICU}$ patients, the CCI was found to be useful to discriminate between survivors and nonsurvivors [15]. They also found that APACHE II system was an excellent predictor. (area $=0.87, \mathrm{SE}=0.04)$. They suggested that CCI could improve prognostic predictions even for critically ill patients. Although they included all patients without age discrimination, they found a significant result for CCI similar to our result.

\section{Limitations}

Limitation of the current study was its retrospective design and heterogenous patient selection. Else, the quality of comorbidity data in retrospective review of medical records or administrative data may be unclear and under-report true incidence. Therefore prospective studies are needed.

\section{CONCLUSION}

Many parameters are used to predict prognosis in ICU patients. The results of the current study revealed that the mortality rates were significantly higher in patients with higher APACHE II scores. The CCI was higher in the non-survivor group and was strongly associated with mortality and LOS in critically ill older patients. Due to its easily available nature, it is appropriate to use it as a prognostic index in critically ill elderly patients.

\section{Conflict of interest}

The authors disclosed no conflict of interest during the preparation or publication of this manuscript.

\section{Financing}

The authors disclosed that they did not receive any grant during conduction or writing of this study.

\section{REFERENCES}

1. Zimmerman JE, Alzola C, Von Rueden KT. The use of benchmarking to identify top performing critical care units: a preliminary assessment of their policies and practices. J Crit Care 2003; 18:76-86.

2. Orsini J, Butala A, Salomon S, Studer S, Gadhia S, Shamian B, et al. Prognostic factors associated with adverse outcome 
among critically ill elderly patients admitted to the intensive care unit. Geriatr Gerontol Int 2015;15:889-94.

3. Talib S, Sharif F, Manzoor S, Yaqub S, Kashif W. Charlson comorbidity index for prediction of outcome of acute kidney injury in critically ill patients. Iran J Kidney Dis 2017;11:115-23. 4. Burch JB, Augustine AD, Frieden LA, Hadley E, Howcroft TK, Johnson R, et al. Advances in geroscience: impact on healthspan and chronic disease. J Gerontol A Biol Sci Med Sci 2014;69 Suppl 1:S1-3.

5. Charlson ME, Pompei P, Ales KL, MacKenzie CR. A new method of classifying prognostic comorbidity in longitudinal studies: development and validation. J Chronic Dis 1987;40:37383.

6. Quan H, Li B, Couris CM, Fushimi K, Graham P, Hider P, et al. Updating and validating the Charlson comorbidity index and score for risk adjustment in hospital discharge abstracts using data from 6 countries. Am J Epidemiol 2011;173:676-82.

7. Núñez JE, Núñez E, Fácila L, Bertomeu V, Llàcer A, Bodí V, et al. [Prognostic value of Charlson comorbidity index at 30 days and 1 year after acu]te myocardial infarction]. Rev Esp Cardiol 2004;57:842-9. [Article in Spanish]

8. Tripathy S, Mishra JC, Dash SC. Critically ill elderly patients in a developing world--mortality and functional outcome at 1 year: a prospective single-center study. J Crit Care 2014;29:474.e7-13.

9. Pisani MA. Considerations in caring for the critically ill older patient. J Intens Care Med 2009;24:83-95.

10. Falsetti L, Viticchi G, Tarquinio N, Silvestrini M, Capeci W, Catozzo V, et al. Charlson comorbidity index as a predictor of inhospital death in acute ischemic stroke among very old patients: a single-cohort perspective study. Neurol Sci 2016;37:1443-8.

11. Chelluri L, Pinsky MR, Donahoe MP, Grenvik A. Long-term outcome of critically ill elderly patients requiring intensive care. JAMA 1993;269:3119-23.

12. Zekry D, Loures Valle BH, Lardi C, Graf C, Michel JP, Gold $\mathrm{G}$, et al. Geriatrics index of comorbidity was the most accurate predictor of death in geriatric hospital among six comorbidity scores. J Clin Epidemiol 2010;63:1036-44.

13. Buntinx F, Niclaes L, Suetens C, Jans B, Mertens R, Van den Akker M. Evaluation of Charlson's comorbidity index in elderly living in nursing homes. J Clin Epidemiol 2002;55:1144-7.

14. Quach S, Hennessy DA, Faris P, Fong A, Quan H, Doig C. A comparison between the APACHE II and Charlson Index Score for predicting hospital mortality in critically ill patients. BMC Health Serv Res 2009;9:129.

15. Poses RM, McClish DK, Smith WR, Bekes C, Scott WE. Prediction of survival of critically ill patients by admission comorbidity. J Clin Epidemiol 1996;49:743-7 